\title{
Two electron dynamics in photodetachment
}

\author{
D. Hanstorp, G. Haeffler, A. E. Klinkmüller, U. Ljungblad \\ Department of Physics, Göteborg University and \\ Chalmers University of Technology $A B$ \\ SE-412 96 Göteborg, Sweden \\ U. Berzinsh \\ Department of Spectroscopy, University of Latvia, \\ LV 1586, Riga, Latvia \\ I. Yu. Kiyan \\ Russian Academy of Sciences, General Physics Institute, \\ 117 942 Moscow, Russia \\ D. J. Pegg \\ Department of Physics, University of Tennessee, Knoxville, \\ TN 37996 USA
}

September 3, 2021

\begin{abstract}
We present the results of experimental studies of photon-negative ion interactions involving the dynamics of two electrons. Resonances associated with doubly excited states of $\mathrm{Li}^{-}$and $\mathrm{He}^{-}$have been observed using laser photodetachment spectroscopy. Total and partial photodetachment cross sections have been investigated. In the former case, the residual atoms are detected irrespective of their excitation state, while in the latter case only those atoms in specific states are detected. This was achieved by the use of a state selective detection scheme based on the resonant ionization of the residual atoms. In addition, in the case of $\mathrm{Li}^{-}$ photodetachment, the threshold behavior of the $\operatorname{Li}\left(2^{2} \mathrm{P}\right)+\mathrm{e}^{-}(\mathrm{ks})$ partial cross section has been used to accurately measure the electron affinity of
\end{abstract} Li. 


\section{Introduction}

We present results of recent studies of the photodetachment of few-electron atomic negative ions. The motivation for these measurements is to better understand the general problem of correlated motion of particles in many-body systems. Correlated motion plays an important role in several fields of physics. In nuclear physics, for example, it manifests itself in halo nuclei, in which the two outermost neutrons contribute to the formation of very diffuse nuclei [1]. In the case of atomic systems, it is known that negative ions, in particular, exhibit an enhanced sensitivity to electron correlation effects due to the suppression of the normally dominant Coulomb force of the core. This sensitivity is further increased in the case of doubly excited states of negative ions where, in general, the outermost electron moves in the field of an excited atom. Such an electron is bound by short range polarization forces, in contrast to the long range Coulomb force characteristic for the binding of atoms and positive ions. The more symmetric the two electron excitation, the more highly correlated the state. A highly excited pair of electrons are no longer independent, rather they begin to move collectively. The autodetaching decay of doubly excited states is manifested as resonances in photodetachment cross sections. Such resonances arise from an interference between two paths to the same final continuum state: direct photodetachment and resonant photodetachment via the intermediate doubly excited state. The results of experiments on negative ions, and particularly those involving resonances associated with excited negative ions, therefore provide sensitive tests of the ability of theory to go beyond the independent electron model.

The prototypical atomic negative ion is $\mathrm{H}^{-}$. Here, two electrons move in the field of a point core, the proton. In general, if both electrons are excited the outer electron moves in the field of an excited $\mathrm{H}$ atom consisting of the nucleus and the inner electron. Since this permanent dipole field differs intrinsically from the long range Coulomb field, the series of resonances will not be strictly Rydberg in nature. Electron correlation becomes more enhanced as the level of excitation increases since the excited $\mathrm{H}$ atom becomes more easily polarizable. Resonances representing the decay of such doubly excited states appear in the photodetachment cross section below the excited $\mathrm{H}$ atom thresholds. Double excitation of the $\mathrm{H}^{-}$ion has been studied extensively both experimentally [2] and theoretically [3]. It is clear that these highly correlated states are not well represented by the independent electron model. Instead, new quantum number labels have been introduced that describe the collective motion of the electron pair 涵. We have chosen to study the two electron dynamics in $\mathrm{He}^{-}$and $\mathrm{Li}^{-}$ions since they represent the logical extension of the work on the $\mathrm{H}^{-}$ion. In the case of both $\mathrm{He}^{-}$and $\mathrm{Li}^{-}$, the two active electrons move in the field of a finite core. $\mathrm{He}^{-}$and $\mathrm{Li}^{-}$ions are therefore simple systems for investigating few-particle interactions since the core, in each case, is finite but small. The spherically symmetric closed core of $\mathrm{Li}^{-}$might be expected to approximate the $\mathrm{H}^{-}$ion somewhat better than the open core of $\mathrm{He}^{-}$. The presence of a core will serve 
to lift the degeneracy of the excited state thresholds characteristic of the $\mathrm{H}$ atom. Doubly excited states will then be bound in the shorter range field of an induced dipole and not a permanent dipole, as is the case for $\mathrm{H}^{-}$. As a consequence, one expects the photodetachment spectra of these ions to differ from that of the $\mathrm{H}^{-}$ ion, at least at low-to-intermediate levels of excitation. For example, the resonances should be broader since the lifting of the threshold degeneracy results in a larger number of continua available for autodetachment [5, 6]. It is also not clear whether the quantum numbers and propensity rules developed to describe the $\mathrm{H}^{-}$spectra will remain valid to the same extent in the case of the $\mathrm{He}^{-}$and $\mathrm{Li}^{-}$spectra. Of course, as the level of excitation increases the role played by the core will become less significant and the spectra, particularly that of $\mathrm{Li}^{-}$, should appear more and more like that of $\mathrm{H}^{-}$. In fact, the $\mathrm{Li}^{-}$ion can be considered as the "poor man's $\mathrm{H}^{-}$ ion" in the sense that at high levels of excitation their spectra should be similar. The doubly excited states of $\mathrm{Li}^{-}$are, however, far more accessible. In both $\mathrm{Li}^{-}$and $\mathrm{He}^{-}$, high lying states can be studied in high resolution experiments using tunable dye lasers. Corresponding states in the $\mathrm{H}^{-}$ion, however, have excitation energies about an order of magnitude larger and therefore remain relatively inaccessible.

\section{Method}

\subsection{Total Cross Sections}

The total photodetachment cross section describes the probability that an electron is detached from a negative ion following the absorption of a photon, regardless the excitation state of the residual atom or the energy or direction of the emitted electron. A total cross section is the sum of partial cross sections for detachment into each of the energetically allowed continua. This is illustrated in Fig. 1. Here we show the three possible channels accessible to a doubly excited state of $\mathrm{Li}^{-}$that lies just below the $\operatorname{Li}\left(3^{2} \mathrm{P}\right)$ detachment threshold. The total cross section may be determined by monitoring the production of either the detached electrons or the neutral atoms left following photodetachment. In ion beam experiments the residual atoms can be efficiently collected in the forward direction since they are moving with the same velocity as the beam ions. In the present experiments we measure the yield of the residual atoms produced when a beam of negative ions is merged collinearly with a beam of pulsed, tunable laser radiation. Studies of double excitation of negative ions demand both high sensitivity and energy resolution as a consequence of the low production cross sections and the high density of resonances below highly excited thresholds. The choice of a collinear geometry has two major advantages over a crossed beam geometry. First, the interaction volume can be made at least two orders of magnitude larger, resulting in a more efficient production of the doubly excited

states. Second, kinematic compression [7] of the longitudinal velocity distribution of the ions significantly improves the energy resolution of the photodetachment spectra. 


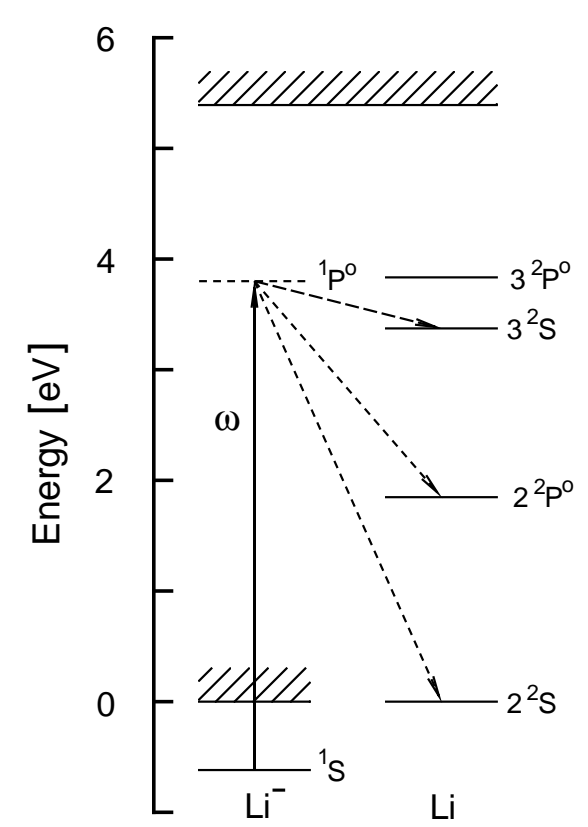

Figure 1: Partial energy level diagram for the $\mathrm{Li}^{-} / \mathrm{Li}$ systems showing the autodetaching decay of a doubly excited state of $\mathrm{Li}^{-}$into different continua. Each of the three continuum channel is characterized by the state that the residual $\mathrm{Li}$ atom is left in following photodetachment via the doubly excited state. A particular decay channel is isolated by state selectively detecting the excited residual atom.

Typically, doubly excited states autodetach predominantly to just a few continua and the associated resonance structure modulates the corresponding partial cross sections. A problem arises in total cross section measurements as high lying doubly excited states are accessed. Resonance structure in total cross sections tends to "wash out" as many partial cross sections with little or no structure are added to those that exhibit well modulated structure. Under such conditions, it becomes necessary to isolate a particular continuum channel and study resonances in the corresponding partial cross section. Another problem arises if one attempts to study the total cross section for the photodetachment of metastable negative ions such as $\mathrm{He}^{-}$. In this case a large neutral atom background arises from autodetachment of the unstable ions in their ground state. To avoid these two problems, we have chosen to selectively ionize the residual atoms and detect positive ions in the presence of a much smaller background. By use of this state selective detection scheme, we hence study partial cross sections instead of total cross sections.

\subsection{Partial Cross Sections}

A partial photodetachment cross section describes the probability that an electron is detached from a negative ion into a particular continuum state. Each decay channel is characterized by the excitation state of the residual atom and the energy and angular momentum of the detached electron. A particular channel can therefore be identified by either measuring the energy of the detached electron, as in photoelectron spectroscopy, or by state selectively detecting the residual atom. We have 
chosen the latter alternative in the present work. The excitation state of the residual atom can be determined by the use of the well established technique of Resonance Ionization Spectroscopy (RIS). This sensitive and selective technique was developed more than 20 years ago [8] but it has only recently been applied to the study of negative ions [9]. The use of a collinear beam geometry combined with the resonant ionization of the residual atoms presents several distinct advantages over photoelectron spectroscopy in which the detached electrons are energy analyzed, invariably using a crossed beam geometry. First, the positive ions can be collected far more efficiently than photoelectrons since they move in one direction only, the ion beam direction. In contrast, the electrons are ejected over a distribution of angles and therefore only a small fraction can be collected within the finite acceptance angle of a detector. Second, the background associated with the detection of positive ions is generally far smaller than that associated with the detection of photoelectrons. Both of these advantages enhance the sensitivity of state selective measurements on the residual atoms. On the other hand, the major advantage of photoelectron spectroscopy is that all energetically allowed photodetachment channels can be isolated and measured simultaneously. Another attribute is that the angular distribution of the detached electrons can be measured. This additional information provides further insight into the photodetachment process. In order to extract information on the parameters of a resonance one must fit a parameter function that describes the interaction of a doubly excited state with continua to the experimental data. The most commonly used form is the Beutler- Fano profile [10]

$$
\sigma(E)=a+\frac{(q+\epsilon)^{2}}{1+\epsilon^{2}}
$$

with,

$$
\epsilon=2 \frac{\left(E-E_{0}\right)}{\Gamma}
$$

where $E$ is the photon energy, $E_{0}$ and $\Gamma$ are the position and width of the resonance, $q$ is the shape parameter, $a$ is the background and $b$ is the amplitude of the resonance. This form is strictly only valid for the treatment of resonance structure in a total cross section. It can, however, be used to parametrize resonances in a partial cross section if, as in the present case, only the positions and widths of the resonances are required. Starace has extended the Beutler-Fano treatment to cover the case of partial cross sections [11]. The form that Starace has proposed can be written as

$$
\begin{aligned}
\sigma(E)=a+\frac{b}{1+\epsilon^{2}}\left[\epsilon^{2}+2 \epsilon(q \Re(\alpha)-\Im(\alpha))+1-2 q \Im(\alpha)\right. & \\
& \left.-2 \Re(\alpha)+\left(q^{2}+1\right)|\alpha|^{2}\right]
\end{aligned}
$$


where the parameters have the same significance as in the Beutler-Fano formula. In addition a complex parameter, $\alpha$, is introduced to describe the branching into a particular channel.

\subsection{Experimental Procedure}

A detailed description of the interacting beams apparatus used in the present work can be found elsewhere 12]. In short, positive ions are extracted from a plasma- type ion source and accelerated to beam energies that can be varied between $2.5 \mathrm{keV}$ and $5 \mathrm{keV}$. Negative ions are produced in the beam by double sequential charge exchange in a cesium vapor. A pair of electrostatic quadrupole deflectors (QD1,2) is used to direct the negative ion beam into and out of the path of the laser beams, as shown schematically in Fig. 2. The ion-laser interaction region is defined by two apertures (AP) of diameter $3 \mathrm{~mm}$ placed $0.5 \mathrm{~m}$ apart. The amount of negative ions remaining after passage through the interaction region is monitored for normalization purposes using a Faraday cup (FC) placed adjacent to the second quadrupole deflector. Neutral atoms produced by photodetachment or collisional detachment are unaffected by the electric field in the second quadrupole. They enter the neutral atom detector (ND) situated downstream of the second quadrupole deflector. Here, the impact of the fast beam atoms on a quartz plate (CG) produces secondary electrons that are efficiently detected using a channel electron multiplier (CEM). The plate is coated with a few atomic layers of platinum in order to prevent charge build-up. The transparency of the plate makes it possible to direct the laser beams both parallel and anti-parallel with respect to the ion beam. By performing both co- and counter-propagating experiments, it is possible to correct for the Doppler shift to all orders. A serious background source with this arrangement, however, is associated with photoelectrons generated on the plate by pulsed UV radiation. This contribution is greatly reduced by modulating the bias on a grid placed between the glass plate and the CEM, as described in detail in 13. This detector has been shown to work efficiently for laser wavelengths down to $250 \mathrm{~nm}$.

The third detector (PD) in the interaction chamber is used to detect positive ions in the state selective measurements. It is situated adjacent to the second quadrupole, opposite the Faraday cup used to monitor the negative ion current. It operates in the same way as the neutral atom detector just described. In this case, however, the detector is out of the line of sight of the laser beam and so laser-generated photoelectrons pose no problem. The positive ion detector is used to state selectively detect residual atoms in the partial cross section measurements. The electric field of the second quadrupole is used both to field ionize the Rydberg atoms and to bend the resulting positive ions into the detector. The positive ion background is usually small, the major contribution arising from double detachment collisions of negative ions with the residual gas in the interaction chamber. This contribution is minimized by a combination of a good vacuum (approximately $10^{-9}$ mbar) and a pair of deflection 


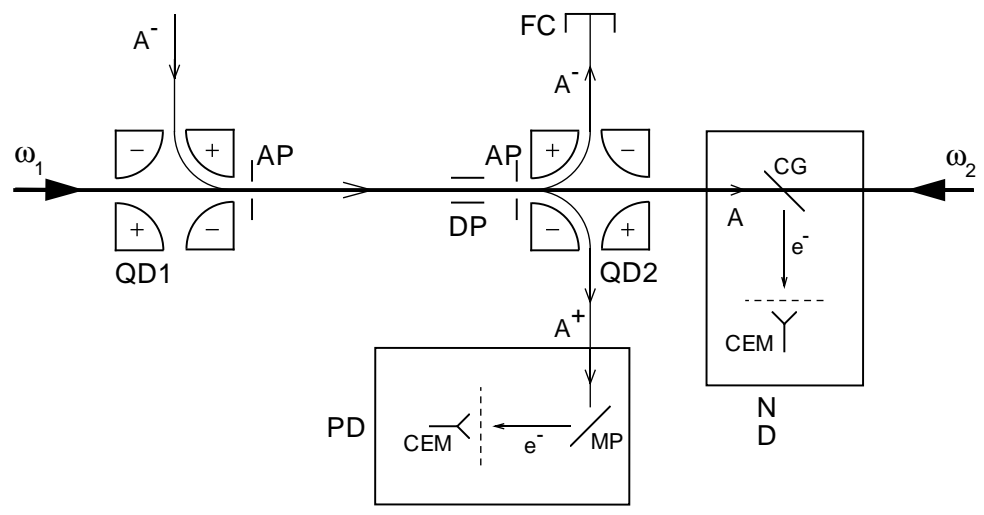

Figure 2: Portion of the collinear laser-ion beam apparatus. QD1,2, electrostatic quadrupole deflectors; CEM, channel electron multiplier; DP, deflection plates; PD, positive ion detector; FC, Faraday cup; ND, neutral particle detector; CG, conducting glass plate; AP, aperture; MP, metal plate; A, any element. The distance between QD1 and QD2 is approximately $0.5 \mathrm{~m}$.

plates (DP) positioned just prior to the entrance of the second quadrupole. The transverse electric field is insufficient to field ionize the atoms prepared in the highly excited Rydberg states but it does serve to sweep out of the beam any positive ions produced upstream in collisional events. This procedure substantially reduces the collisional detachment background since only atoms left in high lying Rydberg states will contribute to the background. Of course, this simultaneously hinders the negative ions from reaching the Faraday cup. The deflection plates are therefore switched off periodically to monitor the negative beam intensity.

The laser system used in the experiments consist of two excimer-pumped dye lasers. The dye lasers can produce narrow bandwidth laser radiation of wavelengths between $340 \mathrm{~nm}$ and $850 \mathrm{~nm}$, which can be extended, with the use of second harmonic generation, down to $205 \mathrm{~nm}$. The bandwidth is nominally about $5 \mathrm{GHz}$, but this can be reduced to $1 \mathrm{GHz}$ by use of an intra cavity etalon. The energy resolution in the present experiments is limited solely by the linewidth of the laser. The maximum pulse energy is typically a few $\mathrm{mJ}$ in the visible wavelength region and a few hundred $\mu \mathrm{J}$ in the UV, after doubling. The laser intensity is measured after the passage through the interaction chamber for normalization purposes. The wavelength of the laser light is calibrated by observing well known resonance lines in $\mathrm{Ne}$ or Ar using optogalvanic spectroscopy in hollow cathode lamps.

\section{Results}




\subsection{Total Cross Section Measurements}

The cross section for photodetachment of an electron from the $\mathrm{Li}^{-}$ion has previously been investigated, both experimentally [14] and theoretically [15]. Earlier laser studies have focused on the region around the first and second detachment thresholds. The latter, also called the first excited state threshold, corresponds to the process in which the residual $\mathrm{Li}$ atom is left in the $2^{2} \mathrm{P}$ state following photodetachment. In contrast to the heavier alkali ions [16], the $\mathrm{Li}^{-}$photodetachment cross section shows no resonance structure below the first excited state threshold. It is expected, however, that doubly excited states should appear at higher levels of excitation. The atomic wave function then becomes more extended in space, making it easier for an extra electron to share the attractive force from the nucleus, or equivalently, the atom becomes more polarizable. We therefore initiated a search for doubly excited ${ }^{1} \mathrm{P}^{\mathrm{o}}$ states of $\mathrm{Li}^{-}$, that are bound with respect to the $3^{2} \mathrm{P}$ state of the $\mathrm{Li}$ atom [17]. The results in the energy region $4.2-4.5 \mathrm{eV}$ are shown in Fig. 3. The $\mathrm{Li}$ atom signal is proportional to the total cross section which, in this case, is equal to the sum of the partial cross sections for the three channels shown in Fig. 1. The experimental cross section data are not absolute. The measurements have therefore been normalized to a theoretical curve at the $\operatorname{Li}\left(3^{2} \mathrm{P}\right)$ threshold. The main contribution to the approximately $10 \%$ scatter in the experimental data arises from changes in the spatial overlap of the laser and ion beams that occurred as the laser frequency was scanned. The statistical scatter due to counting of the residual atoms was less than $3 \%$. The experimental data exhibits three significant features. A small narrow dip is seen at $4.456 \mathrm{eV}$, a rather narrow structure is observed at $4.453 \mathrm{eV}$ (labeled b) and finally, a broad resonance is seen to cover essentially the entire energy region between the $\mathrm{Li}\left(3^{2} \mathrm{~S}\right)$ and $\operatorname{Li}\left(3^{2} \mathrm{P}\right)$ thresholds (labeled a).

The small dip at $4.456 \mathrm{eV}$ is a Wigner cusp that arises from the opening of the $\mathrm{Li}\left(3^{2} \mathrm{P}\right)+\mathrm{e}^{-}(\mathrm{ks})$ photodetachment channel (hereafter labeled $3{ }^{2} \mathrm{Pks}$, for brevity) The other two structures are resonances associated with the decay of doubly excited states. These resonances overlap one another as well as the Wigner cusp, making it impossible to extract information on individual positions and widths. The BeutlerFano formula, which is normally used to extract resonance parameters, cannot be applied in this case since it is only valid for isolated resonances. In order to explain the origin of the observed structures in the $\mathrm{Li}^{-}$photodetachment cross section, comparisons with two independent calculated cross sections were made. Lindroth 17] used an approach which combines complex rotation [18] with a discrete numerical basis set [19], whereas Pan et al. [5] employed the R-matrix method. The two theoretical methods yield essentially identical results (see the solid line in Fig. 3). We observe a rather good agreement between theory and experiment over the whole energy region studied. According to Lindroths calculation, the broad structure (a) is identified with an intrashell resonance associated with the symmetrical excitation of the two valence electrons. This resonance was found to be dominated by the $3 \mathrm{p} 3 \mathrm{~d}$ 


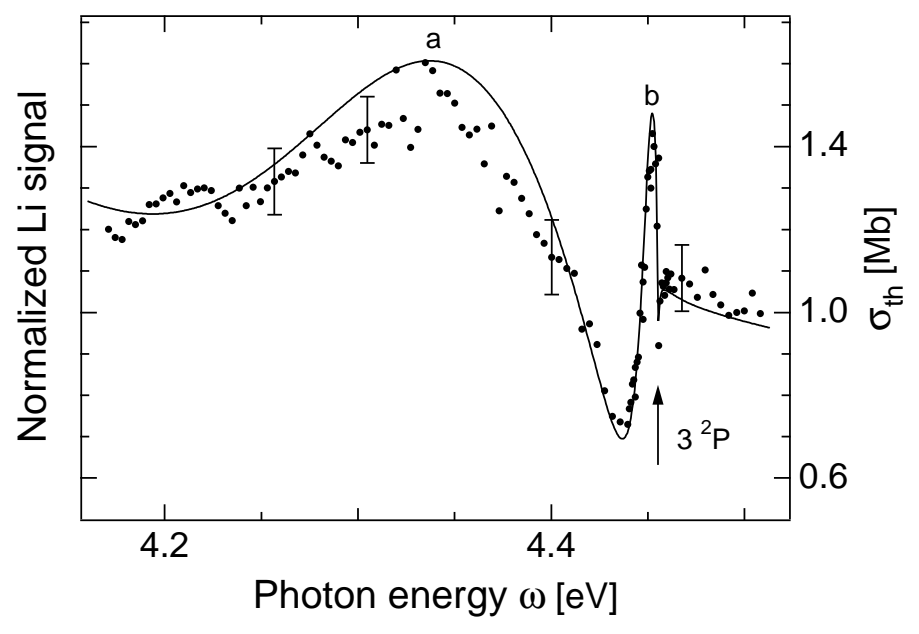

Figure 3: Yield of Li atoms vs. photon energy in the range 4.2-4.5 eV. The Li signal is proportional to the total cross section for the photodetachment of $\mathrm{Li}^{-}$via the $2^{2} \mathrm{Skp}, 2^{2} \mathrm{Pks}, \mathrm{d}$ and $3{ }^{2} \mathrm{Skp}$ channels (see Fig. 1). The experimental data (dots) has been normalized to theory (solid line with scale to the right) at the $3^{2} \mathrm{P}$ threshold.

and $4 \mathrm{~s} 3 \mathrm{p}$ configurations. The narrow structure (b) at approximately $4.453 \mathrm{eV}$ corresponds to several overlapping intershell resonances in which the valence electrons are asymmetrically excited.

\subsection{Partial Cross Section Measurements}

The state selective detection scheme based on RIS has been applied to isolate specific autodetaching channels for doubly excited states in both $\mathrm{He}^{-}$and $\mathrm{Li}^{-}$. Here we present the results of recent measurements of the positions and widths of doubly excited states which appear as resonances in partial cross sections below certain excited state thresholds. The $1 \mathrm{~s} 3 \mathrm{~s} 4 \mathrm{~s}^{4} \mathrm{~S}$ state in $\mathrm{He}^{-}$, for example, lies just below the $\mathrm{He}\left(3^{3} \mathrm{~S}\right)$ threshold. A Feshbach resonance associated with the autodetaching decay of this state was observed in both the $\mathrm{He}\left(1 \mathrm{~s} 2 \mathrm{~s}{ }^{3} \mathrm{~S}\right)+\mathrm{e}(\mathrm{ks})$ and $\mathrm{He}\left(1 \mathrm{~s} 2 \mathrm{p}{ }^{3} \mathrm{P}\right)+\mathrm{e}(\mathrm{kp})$ partial cross sections (hereafter labeled $2^{3} \mathrm{Sks}$ and $2^{3} \mathrm{Pkp}$ ) [20]. Similarly, in the case of $\mathrm{Li}^{-}$, resonances due to doubly excited states below the $\mathrm{Li}\left(4^{2} \mathrm{P}\right)$ and $\operatorname{Li}\left(5^{2} \mathrm{P}\right)$ thresholds were observed in the $3^{2} \mathrm{Skp}$ partial cross section [21]. The state selective detection scheme has also been used to study the threshold behavior of the $2^{2} \mathrm{Pks}$ partial cross section. This technique enabled Haeffler et al. 22] to accurately measure the electron affinity of Li. 


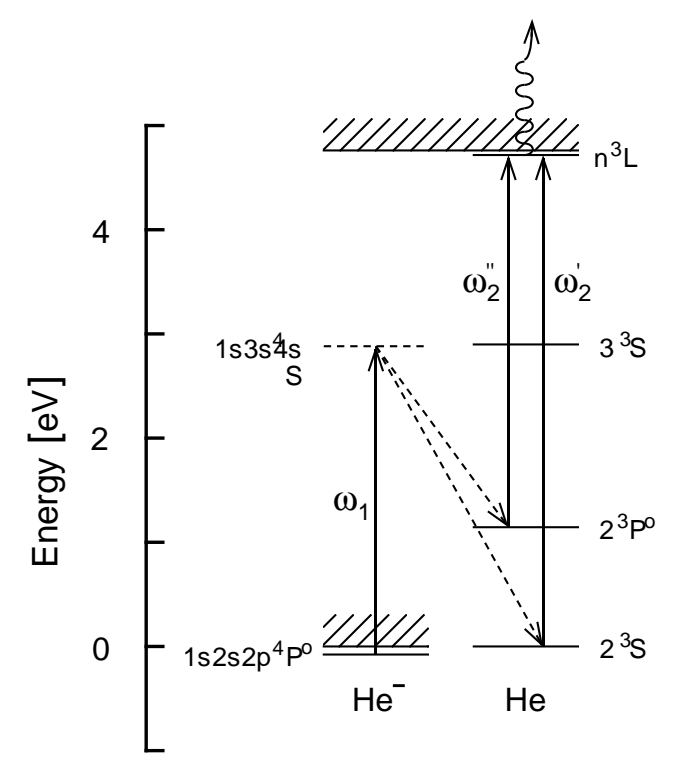

Figure 4: Partial energy level diagram for the $\mathrm{He}^{-} / \mathrm{He}$ systems. Solid lines represent photoexcitation, the wavy line field ionization and dashed lines autodetachment. The $1 \mathrm{~s} 3 \mathrm{~s} 4 \mathrm{~s}{ }^{4} \mathrm{~S}$ state is produced by absorption of photons of frequency $\omega_{1}$. The $2^{3}$ Sks and $2^{3} \mathrm{Pkp}$ decay channels were isolated by selectively detecting the residual $\mathrm{He}\left(2^{3} \mathrm{~S}\right)$ and $\mathrm{He}\left(2^{3} \mathrm{P}\right)$ atoms using RIS (laser $\omega_{2}^{\prime}$ and $\omega_{2}^{\prime \prime}$, respectively).

\subsubsection{Doubly Excited States of $\mathrm{He}^{-}$}

Doubly excited states of $\mathrm{He}^{-}$of doublet symmetry have been observed in studies of electron impact on He [23]. In contrast, data on quartet states are sparse. Selection rules on photoexcitation from the ${ }^{4} \mathrm{P}^{\mathrm{o}}$ ground state limit excited state production to those of ${ }^{4} \mathrm{~S},{ }^{4} \mathrm{P}$ and ${ }^{4} \mathrm{D}$ symmetry. Recently, the $\mathrm{He}^{-}$photodetachment cross section below $4 \mathrm{eV}$ has been calculated by $\mathrm{Xi}$ and Froese Fischer [24]. They predicted a number of resonances associated with the decay of doubly excited quartet states. Bae and Peterson 25] observed a $\left(1 \mathrm{~s} 2 \mathrm{p}^{2}{ }^{4} \mathrm{P}\right)$ shape resonance in the total photodetachment cross section of $\mathrm{He}^{-}$just above the $\mathrm{He}\left(2^{3} \mathrm{P}\right)$ threshold. The parameters of this resonance were subsequently measured more accurately by Walter et al. [26]. We recently embarked on a program aiming to investigate doubly excited states in $\mathrm{He}^{-}$below the $\mathrm{He}(n>2)$ thresholds. The first measurement involved the $1 \mathrm{~s} 3 \mathrm{~s} 4 \mathrm{~s}{ }^{4} \mathrm{~S}$ state that lies below the $\mathrm{He}\left(3^{3} \mathrm{~S}\right)$ threshold. The decay of this state was observed as a Feshbach resonance in both the $2^{3} \mathrm{Sks}$ and the $2^{3} \mathrm{Pkp}$ partial cross sections.

Fig. 4 shows selected energy levels of the $\mathrm{He}^{-} / \mathrm{He}$ systems. The $1 \mathrm{~s} 3 \mathrm{~s} 4 \mathrm{~s}^{4} \mathrm{~S}$ state of $\mathrm{He}^{-}$is situated just below the $\mathrm{He}\left(3^{3} \mathrm{~S}\right)$ threshold. This state, which is excited with laser $\omega_{1}$, rapidly autodetaches via the $2^{3} \mathrm{Sks}$ and $2^{2} \mathrm{Pkp}$ channels. Following the decay, the residual He atom will be left in either the $2{ }^{3} \mathrm{~S}$ or $2{ }^{3} \mathrm{P}$ excited states. Two different laser frequencies $\omega_{2}^{\prime}$ and $\omega_{2}^{\prime \prime}$ were applied separately in the resonance ionization scheme used to monitor the population of the $2{ }^{3} \mathrm{~S}$ and $2{ }^{3} \mathrm{P}$ states. The frequency was chosen to induce a transition between the $2^{3} \mathrm{~S}$ and the $24^{3} \mathrm{P}$ states of the He atom, when photodetachment into the $2^{3} \mathrm{Sks}$ channel was studied. The frequency $\omega_{2}^{\prime \prime}$ induced a resonance transition between the $2^{3} \mathrm{P}$ and $26^{3} \mathrm{D}$ states of $\mathrm{He}$, when photodetachment into the $2^{3} \mathrm{Pkp}$ channel was studied. The population 
of both the high lying Rydberg states were efficiently depleted by the electric field of the second quadrupole deflector and $\mathrm{He}^{+}$ions thus produced were recorded as a function of frequency of laser $\omega_{1}$. The output of laser $\omega_{1}$ was attenuated to avoid saturation of the photodetachment process and the detector.

Fig. 5 shows how the $\mathrm{He}^{+}$signal varies as a function of the laser frequency $\omega_{1}$ in the vicinity of the resonance. The signal is proportional to the partial cross sections for photodetachment via the $2{ }^{3} \mathrm{Sks}$,d channels (Fig. 5a) and the $2{ }^{3} \mathrm{Pkp}$ channel (Fig. 5b). Selection rules forbid the autodetachment of the $1 \mathrm{~s} 3 \mathrm{~s} 4 \mathrm{~s}{ }^{4} \mathrm{~S}$ state via the $2{ }^{3} \mathrm{Skd}$ channel in the LS-coupling approximation and so in Fig. 5 (a) this channel contributes to the background only (we do not resolve the degenerate s- and d-wave channels). According to recent calculations of Xi and Froese Fischer 27] the cross section for the d-wave channel remains nearly constant at $6 \mathrm{Mb}$ across the resonance, while the s-wave channel has a peak value of $20 \mathrm{Mb}$ on the resonance. The difference in the level of the background in Fig. 5(a) and Fig. f (b) arises essentially from the aforementioned d-wave channel. The background in Fig. 5 (b), and that remaining in Fig. 5 (a) after the d-wave contribution has been subtracted, arises from several sources. The two most significant background processes that produce $\mathrm{He}^{+}$ ions, indirectly from $\mathrm{He}^{-}$ions, are collisional single detachment and photodetachment processes by laser $\omega_{2}^{\prime}\left(\omega_{2}^{\prime \prime}\right)$ that populate the $\operatorname{He}\left(2{ }^{3} \mathrm{~S}, 2{ }^{3} \mathrm{P}\right)$ states. Once these states are formed by background processes, they will be resonantly ionized by the laser frequencies $\omega_{2}^{\prime}$ and $\omega_{2}^{\prime \prime}$, respectively, and the resulting $\mathrm{He}^{+}$ions will be indistinguishable from the signal. The $\mathrm{He}^{+}$ions produced directly in double detachment events were essentially eliminated in our experiment by the use of a pair of deflection plates placed just upstream of the second quadrupole deflector (see Fig. 2). The low pressure in the interaction chamber is essential to reduce the background due to collisional detachment processes. In addition, the output of the laser at frequencies $\omega_{2}^{\prime}$ and $\omega_{2}^{\prime \prime}$ was attenuated to reduce the background arising from the photodetachment of $\mathrm{He}^{-}$at these frequencies.

Table 1: A comparison of experimental and theoretical values for the positions and widths of resonant states in $\mathrm{He}^{-}$and $\mathrm{Li}^{-}$relative to the ground state of the negative ion.

\begin{tabular}{llll}
\hline \hline Resonance & \multicolumn{1}{c}{$E_{0}(\mathrm{eV})$} & $\Gamma(\mathrm{eV})$ & Reference \\
\hline $\mathrm{He}^{-}\left({ }^{4} \mathrm{~S}\right)$ & $2.959255(7)$ & $0.00019(3)$ & Experiment [20] \\
& 2.95907 & 0.00019 & Theory [24] \\
$\mathrm{Li}^{-}(\mathrm{c})$ & $5.1132(4)$ & $0.0074(5)$ & Experiment [21] \\
& 5.115 & 0.0073 & Theory [6] \\
$\mathrm{Li}^{-}(\mathrm{d})$ & $5.1234(4)$ & $0.0076(11)$ & Experiment [21] \\
& 5.126 & 0.0099 & Theory [6] \\
\hline \hline
\end{tabular}


continued from previous page

\begin{tabular}{lccl}
\hline \hline Resonance & $E_{0}(\mathrm{eV})$ & $\Gamma(\mathrm{eV})$ & Reference \\
\hline $\mathrm{Li}^{-}(\mathrm{j})$ & $5.4485(1)$ & $0.0018(1)$ & Experiment [21] \\
$\mathrm{Li}^{-}(\mathrm{k})$ & $5.4508(1)$ & $0.0073(3)$ & Experiment [21] \\
\hline \hline
\end{tabular}

The resonance energy and width are obtained by fitting the data shown in Fig. to both a Beutler-Fano profile (11) and to the form proposed by Starace (2). As expected, the values of $E_{0}$ and $\Gamma$ were the same using both parametrizations. In each case, the measurement was performed with co-propagating and counter- propagating laser $\left(\omega_{1}\right)$ and ion beams to eliminate the Doppler effect associated with the fast moving ions. The data shown in Fig. 5, for instance, have been recorded with co-propagating laser $\left(\omega_{1}\right)$ and ion beams. The fit to these data yields the blueshifted resonance position, $E_{0}^{\mathrm{b}}$. The measurement was repeated with counter- propagating beams to yield the red-shifted resonance position, $E_{0}^{\mathrm{r}}$. The resonance position, corrected for the Doppler effect to all orders, is given by the geometric mean, $E_{0}=\sqrt{E_{0}^{\mathrm{b}} E_{0}^{\mathrm{r}}}$, of the red- and blue-shifted energies. Table 11 compares the present measurement of the resonance position and width with the result of a recent calculation of $\mathrm{Xi}$ and Froese Fischer [24]. One can see that the present results are in good agreement with theory.

\subsubsection{Doubly Excited States in Li-}

The method of studying partial photodetachment cross sections based on the selective detection of the residual atoms has also been used to investigate resonances associated with the decay of doubly excited states of $\mathrm{Li}^{-}$. Fig. 6 illustrates a state selective detection scheme designed, in this case, to isolate the $3{ }^{2} \mathrm{Skp}$ partial cross section in $\mathrm{Li}^{-}$photodetachment. A laser of frequency $\omega_{1}$ excites the negative ion into a ${ }^{1} \mathrm{P}$ doubly excited state that is embedded in several continua. This transient state subsequently autodetaches producing a free electron and a neutral Li atom which, in general, is excited. In the case shown in the figure, the quasi-discrete state is embedded in six continua i.e. there are six possible decay channels that are energetically accessible. These channels are characterized by the residual Li atom being left in $2^{2}$ $\mathrm{S}, 2^{2} \mathrm{P}, 3^{2} \mathrm{~S}, 3^{2} \mathrm{P}, 3^{2} \mathrm{D}$ or $4^{2} \mathrm{~S}$ state following detachment. A second laser of frequency $\omega_{2}$ is applied to resonantly photoexcite those Li atoms left in the $3^{2} \mathrm{~S}$ state to the $22{ }^{3} \mathrm{P}$ state. Atoms in such highly excited Rydberg states are efficiently ionized by the electric field of the second quadrupole. The positive ions thus produced can be efficiently detected in the presence of a relatively low background. This procedure isolates the $3{ }^{2} \mathrm{Skp}$ channel.

In Fig. 7, we show the cross section for photodetachment of $\mathrm{Li}^{-}$via the $3^{2} \mathrm{Skp}$ channel over photon energies of approximately 5.04-5.16 eV and 5.29-5.46 eV. These ranges cover the regions below, and including, the $\operatorname{Li}\left(4^{2} \mathrm{P}\right)$ and $\operatorname{Li}\left(5^{2} \mathrm{P}\right)$ thresholds, 

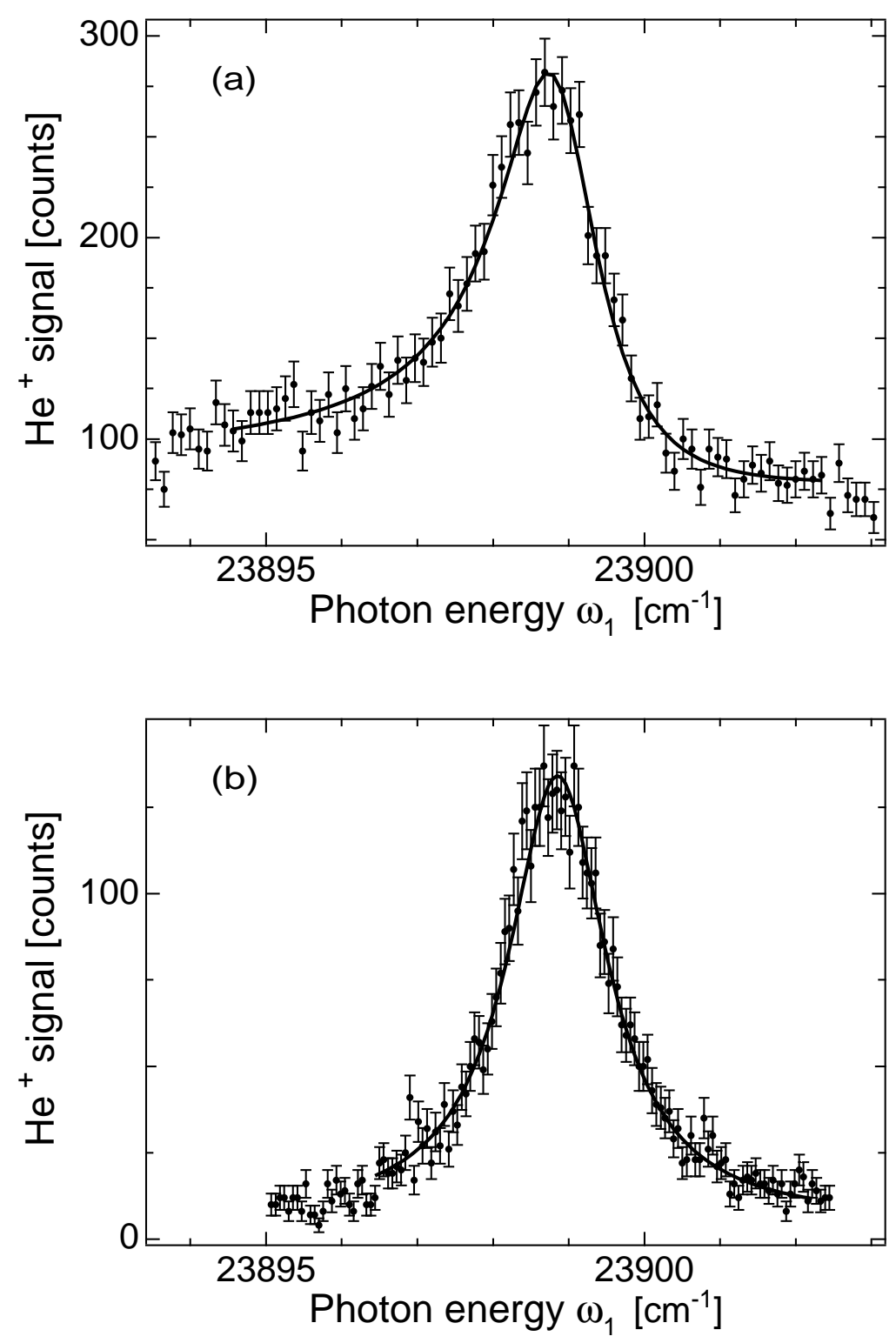

Figure 5: Yield of $\mathrm{He}^{+}$ions vs. photon energy. The $\mathrm{He}^{+}$signal is proportional to the partial cross section for the photodetachment of $\mathrm{He}^{-}$via the $2^{3} \mathrm{Sks}$, d channel (a) and the $2^{3} \mathrm{Pkp}$ channel (b). The solid line represents a fit of the Fano function (11) to the experimental data (dots). 


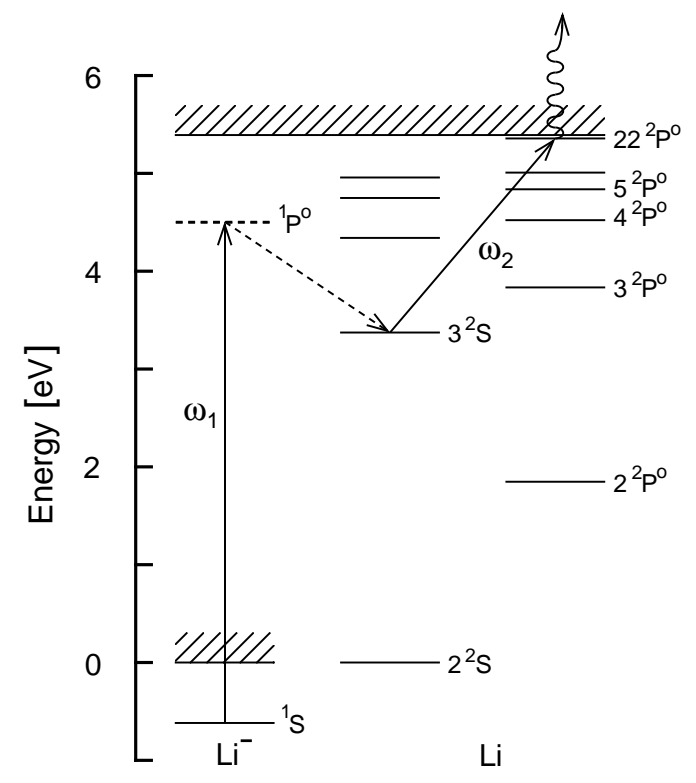

Figure 6: Partial energy level diagram for the $\mathrm{Li} / \mathrm{Li}^{-}$systems. Solid lines represent photoexcitation, the wavy line field ionization and the dashed line autodetachment via the $3^{2}$ Skp channel. Doubly excited states of $\mathrm{Li}^{-}$ are produced by the absorption of photons of frequency $\omega_{1}$. The $3^{2}$ Skp partial cross section is isolated by selectively detecting the residual atoms left in the $3{ }^{2} \mathrm{~S}$ state using RIS (laser $\omega_{2}$ ).

respectively. Several Feshbach "window" resonances are observed to lie below these thresholds. In the figure the present measurements are compared with the result of a recent eigenchannel R-matrix calculation by Pan et al. [28]. The experimental resolution, which is estimated to be about $25 \mu \mathrm{eV}$, is sufficiently high compared to the typical resonance widths that a direct comparison with theory can be made without resorting to deconvolution procedures. At photon energies just below $5.39 \mathrm{eV}$, the ionization energy of $\mathrm{Li}$, we also observed in-board calibration lines arising from the photoexcitation of ground state Li atoms, by laser $\omega_{1}$, to high lying Rydberg states that are subsequently field ionized. It was unnecessary in this case to resort to the use of co- and counter-propagating beams to eliminate the Doppler effect since the resonances in the $\mathrm{Li}^{-}$spectra are much broader than those in the $\mathrm{He}^{-}$spectra. The calibration procedures described above sufficed to determine the resonance position. The cross section measurements are relative. In Fig. $7 \mathrm{a}$ and $7 \mathrm{~b}$, they have been normalized to the theoretical results of Pan et al. [28] by multiplying the data by factors equal to the ratio of the areas under the experimental and theoretical cross section curves between the limits 5.04-5.16 eV and 5.39-5.46 eV, respectively.

Fig. 7 shows that there is generally a good qualitative agreement between experiment and theory in the sense that all the predicted resonances have been observed and no new resonances are apparent. There is also excellent quantitative agreement in the case of the resonant structure below the $\mathrm{Li}\left(4^{2} \mathrm{P}\right)$ threshold, between $5.04 \mathrm{eV}$ and $5.16 \mathrm{eV}$ (Fig. ㄱa). The situation is slightly different, however, for the resonances below the $\operatorname{Li}\left(5^{2} \mathrm{P}\right)$ threshold. Here, theory fails to predict the exact energies and strengths of the resonances. For example, there exists a difference in energy between the measured and calculated resonances ranging from $0-2 \mathrm{meV}$. The size of 

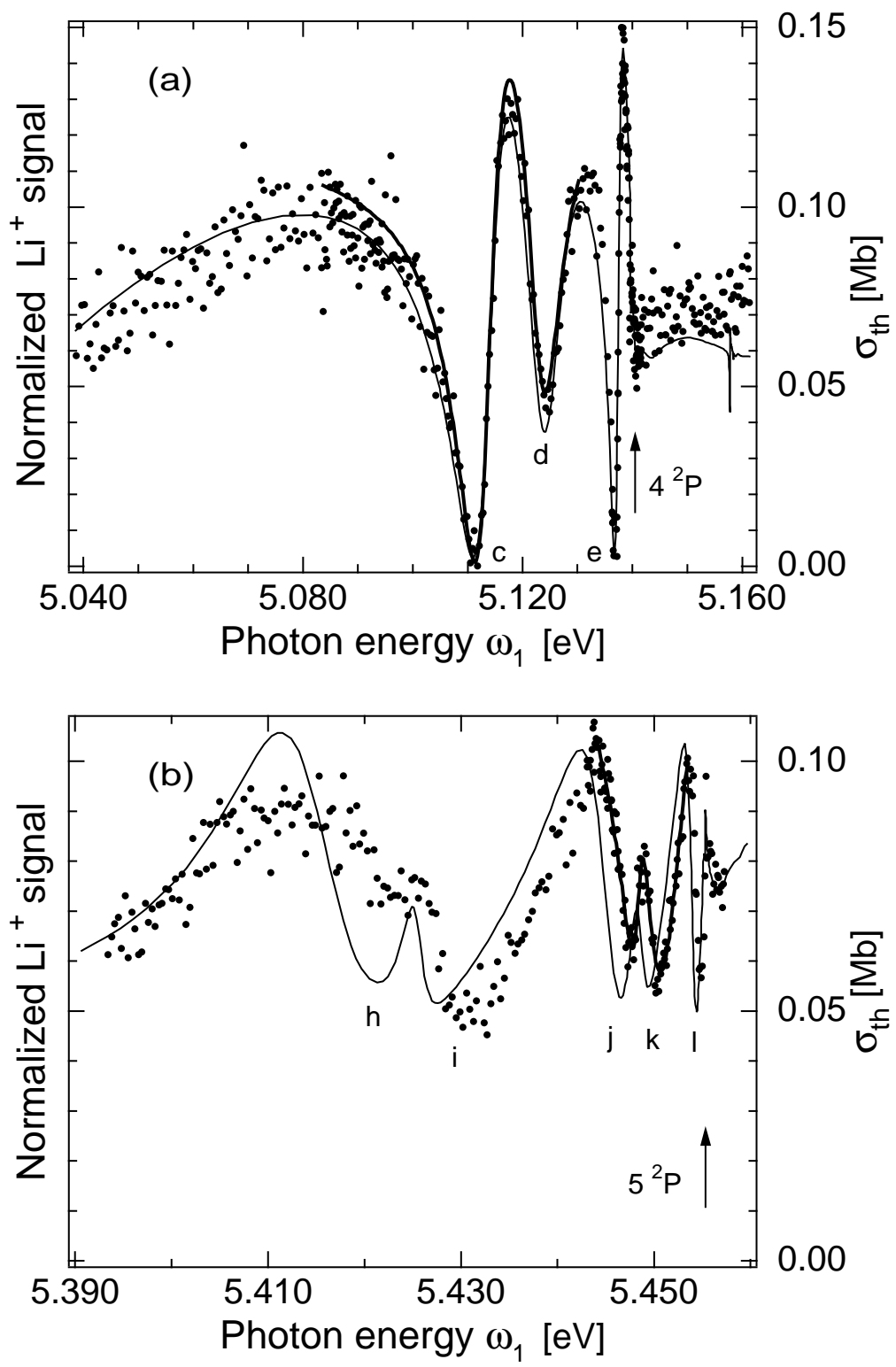

Figure 7: Yield of $\mathrm{Li}^{+}$ions vs. photon energy in the ranges 5.04-5.16 eV (Fig. 7a) and 5.39-5.46 eV (Fig. 7 b). The $\mathrm{Li}^{+}$signal is proportional to the partial cross section for photodetachment of $\mathrm{Li}^{-}$via the $3^{2} \mathrm{Skp}$ channel (see Fig. 6). The experimental data (dots) has been normalized to theory (solid line with scale at the right). The thick solid lines indicate fits of the double Fano formula (3) to the data. 
the discrepancy appears to increase the further the resonance is from the threshold. Experiment and theory overlap, however, close to the threshold. The resonance labeled $\mathrm{h}$ is also seen to be considerably weaker than theoretically predicted, while resonance $\mathrm{j}$ is not as strong as resonance $\mathrm{k}$, contrary to theory. The more recent calculation by Liu and Starace [29] shows, however, an improved agreement with the experimental data.

The window resonances shown in Fig. 7 partially overlap each other, and it is therefore not possible to fit a single Fano profile to each resonance. We have instead fitted a form representing the sum of two Fano profiles to the data in order to extract the energy and width of each resonance pair:

$$
\sigma(E)=a+\sum_{n=1}^{2} b_{n} \frac{\left(q_{n}+\epsilon_{n}\right)^{2}}{1+\epsilon_{n}^{2}}
$$

with

$$
\epsilon_{n}=2 \frac{\left(E-E_{0 n}\right)}{\Gamma}
$$

Here, the index $n$ represent the parameters for each resonance. Using this method we determined the parameters for resonances $c$ and $d$ in Fig. 7a. The measured positions and widths are shown in Table 1, along with corresponding values calculated by Lindroth [6]. There is a good agreement between the experiment and theory in this case. Lindroth's resonance parameters are derived directly from a complex rotation calculation. The R-matrix calculation of Pan et al. [28] did not explicitly yield the resonance parameters and therefore cannot be used for comparison. Since the Fano formula strictly only applies to total cross sections, the values of the $q$ shape parameters are not entirely meaningful in the context of partial cross sections. This parameter is therefore omitted in the table.

We also attempted, unsuccessfully, to include the resonance labeled e in the fitting procedure. In this case it appears that the resonance is prematurely terminated by the opening of the $4^{2} \mathrm{Pks}$ channel. The resonance energies in this case depended strongly on the interval of the fit, which was not the case when the $\mathrm{c}$ and d resonances were treated as a pair. In a similar manner, the double Fano formula was fit to the two resonances labeled $\mathrm{j}$ and $\mathrm{k}$. The parameters obtained from this fit are also included in Table 1. In this case there is no theoretical data available for comparison.

In Fig. 8 we show an extended $3{ }^{2} \mathrm{Skp}$ partial photodetachment cross section which includes measurements below the $\operatorname{Li}\left(5^{2} \mathrm{~S}\right)$ threshold in addition to the data already shown in Fig. $7 \mathrm{~b}$. The data in the range 5.29-5.39 eV represents a relatively low statistics survey scan. It is normalized to the calculation of Pan et al. [28] using the same factor as for the data between 5.39-5.46 eV. It is clear, even from this relatively low quality data, that the two resonances labeled $\mathrm{f}$ and $\mathrm{g}$ are observed at approximately the calculated energies but their measured strengths appear to 


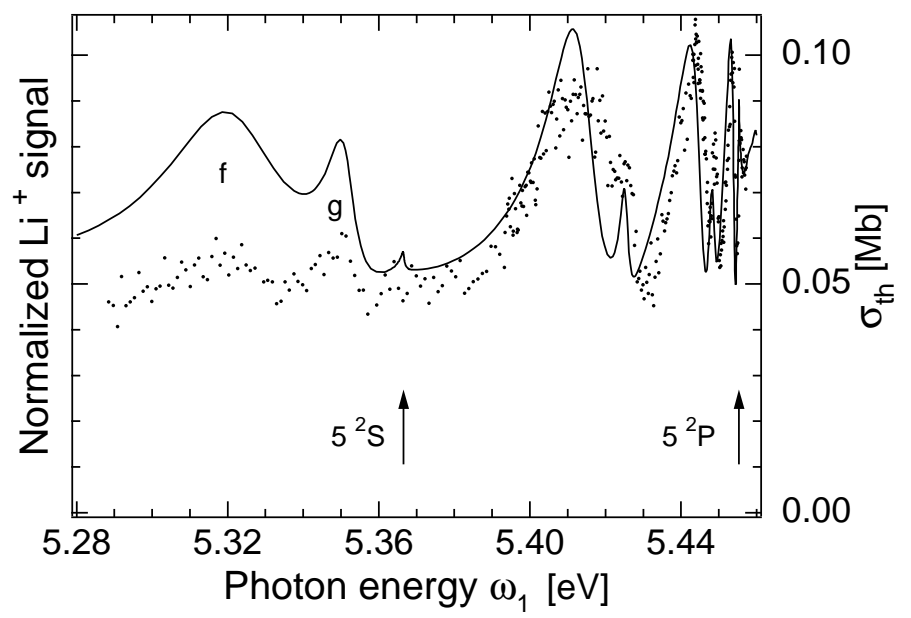

Figure 8: Yield of $\mathrm{Li}^{+}$ions vs. photon energy in the ranges 5.29-5.46 eV (Fig. 7b). $\mathrm{The}^{+}{ }^{+}$signal is proportional to the partial cross section for photodetachment of $\mathrm{Li}^{-}$via the $3{ }^{2} \mathrm{Skp}$ channel (see Fig. 6). The experimental data (dots) has been normalized to theory (solid line with scale at the right).

be weaker than predicted. Presumably, the lowest lying resonance, labeled $\mathrm{f}$, is the intrashell resonance representing symmetric excitation of the two valence electrons.

\subsubsection{Threshold Investigations of $\mathrm{Li}^{-}$}

Wigner [30] predicted that in the vicinity of a threshold the photodetachment cross section is represented by

$$
\sigma(E) \sim\left\{\begin{array}{ll}
\left(E-E_{0}\right)^{l+1 / 2} & E \geq E_{0} \\
0 & E<E_{0}
\end{array},\right.
$$

where $E-E_{0}$ is the excess energy above threshold and $l$ is the angular momentum of the outgoing electron. This form has been tested experimentally [31] and is now well established. The range of validity of the Wigner law describing threshold behavior, however, varies from system to system. It is primarily determined by the polarizability of the residual atom [32]. Resonances near the threshold also play a role in limiting the energy region over which the threshold law applies. If the outgoing electron is represented by $l=0$ ( $\mathrm{s}$-wave detachment) the cross section rises with an infinite slope at the threshold. This allows one, in principle, to perform very accurate threshold measurements. If, however, $l \geq 1$ the slope of the cross section is zero at threshold, making high precision measurements much more difficult.

An investigation of the threshold behavior of the photodetachment process in a swave detachment channel, using tunable laser sources, has the potential for yielding 
the most accurate values for electron affinities. It was recently demonstrated [22, 33] that, by state selectively detecting the residual atom following photodetachment, it is possible to determine an electron affinity to very high accuracy for, in principle, almost any element. In such a scheme, the residual atoms are resonantly ionized and the resulting positive ions are detected in the presence of a low background. The excellent signal-to-background ratio in this case produces data of high statistical quality, which is especially valuable in the vicinity of a threshold. In addition, the use of a collinear laser-ion beam geometry results in high energy resolution measurements. Again, this is particularly valuable near a threshold where the range of validity of the Wigner law is limited. We demonstrated the potential of this technique 22] in a measurement of the electron affinity of Li. The result is about an order of magnitude more accurate than any previous measurement. In the experiment we selectively detected the residual $\mathrm{Li}$ atoms left in the $2^{2} \mathrm{P}$ state following photodetachment via the $2^{2} \mathrm{Pks}$ channel. The sharp onset of production of $\mathrm{Li}^{+}$ions at this s-wave detachment threshold enabled us to easily fit the Wigner law form to the near-threshold data and extrapolate to the threshold value. The data shown in Fig. 9 were taken with counter-propagating laser and ion beams. The measurement was repeated with co-propagating beams. The threshold energy was determined, corrected for the Doppler effect to all orders, from the geometrical mean of the measured blue- and red-shifted threshold energies. The energy of the threshold of the $2^{2} \mathrm{Pks}$ channel was found to be $19888.55(16) \mathrm{cm}^{-1}$. The well known separation of the $2{ }^{2} \mathrm{~S}-$ $2^{2} \mathrm{P}$ states of $\mathrm{Li}$ [34] was subtracted from the threshold energy to yield an electron affinity of $618.049(20) \mathrm{meV}$ using the conversion factor $8065.5410\left(\mathrm{~cm}^{-1} / \mathrm{eV}\right)$.

\section{Conclusions}

The structures of singly excited atoms and positive ions are well described within the independent particle approximation. A large base of experimental data is available for such systems. More loosely bound systems such as negative ions and doubly excited systems in general are, however, more sensitive to electron correlation since here the Coulomb field becomes less dominant. In these cases, the independent electron model begins to break down and collective motion becomes apparent. Experimental data on doubly excited states of negative ions, where correlation effects are of paramount importance, are relatively sparse. Until a few years ago, for example, a systematic investigation of doubly excited states had only been made in the case of the simplest two electron negative ion, $\mathrm{H}^{-}$.

In the present work we have extended the study of double excitation to the $\mathrm{He}^{-}$ and $\mathrm{Li}^{-}$ions which also have two active valence electrons. We have determined the positions and widths of several states in these systems by analyzing resonances in photodetachment cross sections. The measurements were performed under conditions of high sensitivity and resolution since production cross sections are typically 


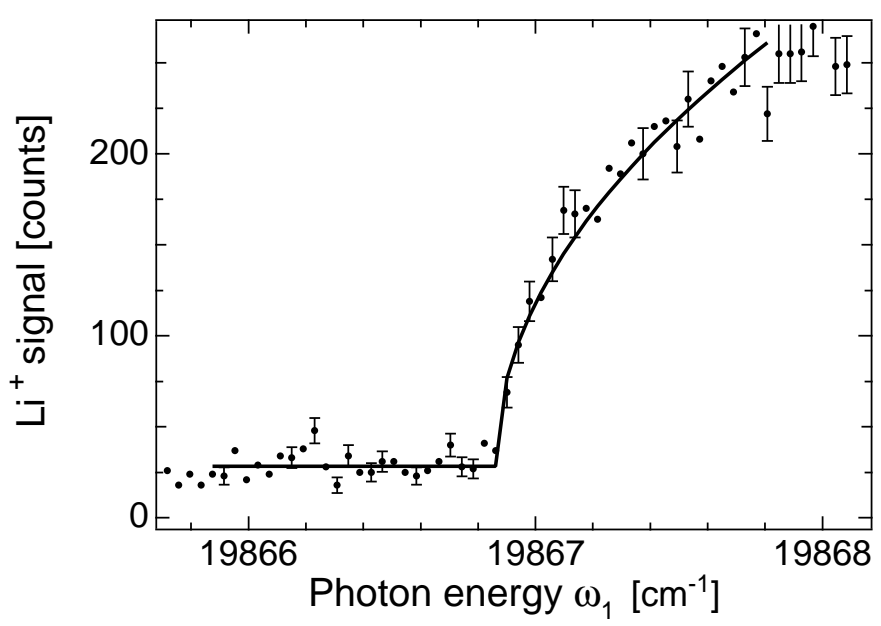

Figure 9: Yield of $\mathrm{Li}^{+}$ions vs. photon energy in the region of the $2{ }^{2} \mathrm{P}$ threshold. The $\mathrm{Li}^{+}$signal is proportional to the partial cross section for photodetachement of $\mathrm{Li}^{-}$via the $2^{2} \mathrm{Pks}$ channel (See Fig. 1). The solid line is a fit of the Wigner law (5) to the data in the range shown. The error bars on selected data points represent the shot noise.

small and resonances are close lying in energy. The sensitivity and resolution were enhanced by combining a collinear laser-ion beam apparatus with the detection of positive ions produced by resonantly ionizing the residual atoms from the photodetachment process. This state selective detection scheme, which is based on the well established technique of Resonance Ionization Spectroscopy, greatly extends the field of negative ion spectroscopy. It is our intention to continue these studies and investigate doubly excited states in $\mathrm{He}^{-}$and $\mathrm{Li}^{-}$and their branching into different continua, all the way to the double detachment limits.

\section{Acknowledgements}

We would like to take the opportunity to acknowledge the support and encouragement that we have received from Professor Ingvar Lindgren throughout this work. As a theorist, his interest in our work most certainly stems from the enhanced role played by electron correlation in negative ion physics. As a former practicing experimentalist, however, we suspect he derives great pleasure from observing how the development of new experimental techniques allows investigations of more and more subtle effects in atomic systems. Thanks Ingvar, we wish you well in your future pursuits. We also thank E. Lindroth, A. F. Starace, C.-N. Liu, J. Xi and C. F. Fischer for fruitful discussions and for providing us with unpublished data. Finan- 
cial support for this research has been obtained from the Swedish Natural Science Research Council (NFR). DJP acknowledge support from the Swedish Institute, The Royal Swedish Academy of Sciences and the US Department of Energy, Office of Basic Energy Sciences, Division of Chemical Sciences. UB acknowledge personal support from the Swedish Institute and, finally, IYK acknowledge support from the Wenner-Gren Center Foundation.

\section{References}

[1] For example, P. G. Hansen, A. S. Jensen and B. Jonson, Annual Review of Nuclear and Particle Science, 45, eds. C. Quigg, V. Lueth and P. Paul (Palo Alto, CA, 1995), p.591.

[2] For example, P. G. Harris et al., Phys. Rev. Lett. 65, 309 (1990); H. C. Bryant et al., Phys. Rev. Lett. 38, 228 (1977); M. E. Hamm et al., Phys. Rev. Lett. 43, 1715 (1979); M. Halka et al., Phys. Rev.A 44, 6127 (1991).

[3] For example, H. R. Sadeghpour and C. H. Greene, Phys. Rev. Lett. 65, 313 (1990); H. R. Sadeghpour, C. H. Greene and M. Cavagnero, Phys. Rev. A 45, 1587 (1992); J. Z. Tang et al., Phys. Rev. A 49, 1021 (1994).

[4] See, for example, J. W. Cooper, U. Fano and F. Pratts, Phys. Rev. Lett. 10, 518 (1963); D. R. Herrick, M. E. Kellman and R. D. Poliak, Phys. Rev. A 22, 1517 (1980); C. D. Lin, Phys. Rev. Lett. 69, 1633 (1992); C. D. Lin, Phys. Rev. A 29, 1019 (1984); C. D. Lin, Adv. At. Mol. Phys. 22, 77 (1986).

[5] C. Pan, A. F. Starace and C. H. Greene, J.Phys. B 27, L137 (1994).

[6] E. Lindroth, Phys. Rev. A 52, 2737 (1995).

[7] S. L. Kaufman, Optics Comm. 17, 309 (1976).

[8] For example, G. S. Hurst, M. G. Payne, S. D. Kramer and J. P. Young, Rev. Mod. Phys. 51, 767 (1979).

[9] P. Balling et al., J. Phys. B. 26, 3531 (1993).

[10] U. Fano, Phys. Rev 124, 1866 (1961).

[11] A. F. Starace, Phys. Rev. A 16, 231 (1977).

[12] U. Ljungblad, Thesis at Gteborg University (1996).

[13] D. Hanstorp, J. Meas. Sci. Technol. 3, 523 (1992). 
[14] J. Dellwo, Y. Liu and D. J. Pegg, Phys. Rev. A 45, 1544 (1992); Y. K. Bae and J. R. Peterson Phys. Rev. A 32, 1917 (1985).

[15] For example, K. T. Chung and P. Fullbright, Phys. Scr. 45, 445 (1992); C. Froese Fischer J. Phys. B. 26, 855 (1993).

[16] For example, T. A. Patterson, H. Hotop, A. Kasdan, D. W. Norcross and W. C. Lineberger, Phys. Rev. Lett. 32, 189 (1974).

[17] U. Berzinsh et al., Phys. Rev. Lett. 74, 4795 (1995).

[18] E. Lindroth, Phys. Rev. A 49, 4473 (1994).

[19] S. Salomonson and P. ster, Phys. Rev. A 40, 5559 (1989).

[20] A. E. Klinkmüller et al., Accepted by Phys. Rev. A.

[21] U. Ljungblad, D. Hanstorp, U. Berzinsh and D. J. Pegg, Phys. Rev. Lett. 77, 3751 (1996).

[22] G. Haeffler et al., Phys. Rev. A. 53, 4127 (1996).

[23] S. J. Buckman and C. W. Clark, Rev. Mod. Phys. 66, 539 (1994).

[24] J. Xi and C. Froese Fischer, Phys. Rev. A 53, 3169 (1996).

[25] J. R. Peterson, Y. K. Bae and D. L. Huestis, Phys. Rev. Lett. 55, 692 (1985).

[26] C. W. Walter, J. A. Seifert and J. R. Peterson, Phys. Rev. A. 50, 2257 (1994).

[27] J. Xi and C. Froese Fischer, private communication.

[28] C. Pan, A. F. Starace and C. H. Greene, Phys Rev A 53, 840 (1996).

[29] A. F. Starace and C.-N. Liu, private communication.

[30] E. P. Wigner, Phys. Rev., 73, 1002 (1948).

[31] H. Hotop and W. C. Lineberger, J. Phys. Chem. Ref. Data 14, 731 (1985).

[32] T. F. O’Malley, Phys. Rev. 137, A1668 (1965).

[33] V. V. Petrunin et al. Phys. Rev. Lett.75, 1911 (1995).

[34] C. J. Sansonetti and B. Richou, Bull. Am. Phys. Soc. 40, 1272 (1995). 